American Journal of Biochemistry and Biotechnology 4 (3): 265-272, 2008

ISSN 1553-3468

(C) 2008 Science Publications

\title{
The Effect of S-Nitroso-N-Acetylpenicillamine and S-Nitroso-Captopril Co-Administered with Cysteine on Blood Glucose Concentration in an Animal Model
}

\author{
${ }^{1}$ Sacha Campbell, ${ }^{1}$ Ruby Alexander-Lindo, ${ }^{2}$ Tara Dasgupta, \\ ${ }^{3}$ Donovan McGrowder and ${ }^{4}$ Lorenzo Gordon \\ ${ }^{1}$ Department of Basic Medical Sciences (Biochemistry Section), \\ ${ }^{2}$ Department of Chemistry, \\ ${ }^{3}$ Department of Pathology, \\ Faculty of Medical Sciences, University of the West Indies, Kingston 7, Jamaica \\ ${ }^{4}$ Department of Medicine, Faculty of Medical Sciences, \\ University of the West Indies, Mona Campus, Kingston, Jamaica
}

\begin{abstract}
The study sought to test the hypothesis that co-administration of SNAP and cysteine or CapSNO and cysteine in normoglycaemic rats may further reduce glucose tolerance. An oral glucose tolerance was carried out and the plasma nitrite concentrations were measured and taken as the biochemical markers of in vivo NO formation. The results showed blood glucose levels were significantly elevated at fasting (F2) and $2.5 \mathrm{~h}$ time intervals of the oral glucose tolerance test on administration of SNAP at $12.5 \mathrm{mg} \mathrm{kg}^{-1}$ body weight (BW) or CapSNO at $12.5 \mathrm{mg} \mathrm{kg}^{-1} \mathrm{BW}$ compared to captopril (12.5 mg kg${ }^{-1} \mathrm{BW}$, control). Co-administration of SNAP and cysteine (30 $\left.\mathrm{mg} \mathrm{kg}^{-1} \mathrm{BW}\right)$, or CapSNO and cysteine enhanced the postprandial hyperglycemic effect at the 2.0 or $2.5 \mathrm{~h}$ time intervals compared with the administration of SNAP or CapSNO only $(\mathrm{p}<0.05)$. The increased postprandial blood glucose concentration was associated with a significant elevation of NO produced from CapSNO and SNAP as assessed by plasma nitrite concentration. Co-administration of cysteine and CapSNO caused a significant increase in total nitrite concentration compared with the administration of only CapSNO $(52.63 \pm 2.69 \mu \mathrm{M}$ vs $32.36 \pm 3.66 \mu \mathrm{M}, \mathrm{p}=0.03)$. The results suggested that in normoglycaemic rats, NO released from SNAP and CapSNO caused a significant hyperglycaemic effect $(\mathrm{p}<0.05)$. This effect was enhanced by cysteine as evidenced by the observed increased total nitrite concentration resulting in exacerbation of the impaired glucose tolerance.
\end{abstract}

Key words: Nitric oxide, s-nitrosothiols-cysteine, s-nitroso-N-acetylpenicillamine, s-nitroso-captopril

\section{INTRODUCTION}

Nitric oxide (NO) is a ubiquitous molecule that is implicated in many physiological processes. It is capable of interacting with many cellular targets, including heme and non-heme iron, thiols, oxygen and superoxide anions ${ }^{[1]}$. Reactions with these targets can result in physiological effects such as the inactivation of guanylate cyclase or the S-nitrosylation of proteins leading to signaling functions ${ }^{[2,3]}$. The reaction of NO with thiols results in the formation of S-nitrosothiols (RSNOs) which act as storage and transport molecules of $\mathrm{NO}^{[4]}$. S-nitrosothiols are present at micro-molar concentrations in the body and are more stable than NO. Some of these compounds are unable to permeate cell membranes due to their hydrophilic nature ${ }^{[5]}$. However studies have shown that S-nitroso-captopril (CapSNO) is able to cross cell membranes ${ }^{[6]}$. The rate of decomposition of RSNOs is increased in the presence of reduced metal ions ${ }^{[7]}$, ascorbic acid $^{[8]}$ and thiols such as glutathione ${ }^{[9]}$ and cysteine ${ }^{[10]}$.

$\mathrm{S}$-nitroso-N-acetylpenicillamine (SNAP) and CapSNO are examples of synthetic RSNOs which have been used extensively in research. S-nitroso-Nacetylpenicillamine is one of the most commonly used S-nitrosothiols in experimental setting, however NO released from SNAP is unpredictable due to its sensitivity to $\mathrm{Cu}(\mathrm{I})$ ions ${ }^{[11]}$. It has been shown to have powerful vasodilatory effect and antiplatelet activity in the cardiovascular system $^{[4]}$ and was found to

Corresponding Author: Sacha Campbell, Department of Basic Medical Sciences, Faculty of Medical Sciences, University of the West Indies, Mona, Kingston 7, Jamaica Tel: +876 - 927-2290 Fax: +876-977-1811 
significantly reduce mean arterial blood pressure in animals $^{[12]}$. However, its use as an antiplatelet agent is limited by its intense vasodilatory and hence hypotensive effects. The half-life of SNAP is $1.15 \mathrm{~h}^{[13]}$ and decomposition of SNAP involves the hemolytic cleavage of the S-NO bond with the release of NO and the formation of an alkyl thiyl radical followed by the combination of two thiyl radicals to give the corresponding disulfide ${ }^{[14]}$.

S-nitroso-captopril is a S-nitroso derivative and it is a hybrid compound of both captopril and $\mathrm{NO}^{[15]}$. As a unique compound, it has properties of both a direct nitrovasodilator and an angiotensin converting enzyme (ACE) inhibitor and is not cross-tolerant with gylceryl trinitrate $(\mathrm{GTN})^{[16]}$. S-nitroso-captopril is water soluble and relatively stable in physiological solution having a half-life of $2.5 \mathrm{~h}$. It activates soluble guanylate cyclase in smooth muscle and platelets, leading to direct, endothelium-independent smooth muscle relaxation and inhibition of platelet aggregation, respectively ${ }^{[16]}$. These properties of CapSNO make it a very interesting pharmacological agent.

Experimental evidence from animal studies suggested that SNAP had a beneficial effect of reducing blood pressure, however this was associated with decreased glucose tolerance ${ }^{[12]}$. We sought to test the hypothesis that co-administration of CapSNO and cysteine or SNAP and cysteine in an animal model may further deteriorate glycemic control. Therefore the study investigates the effects of CapSNO, or SNAP coadministered with cysteine on blood glucose concentration in rats. The total plasma nitrite concentration was measured to determine whether the mode of action is via the release of NO.

\section{MATERIALS AND METHODS}

Animal care-experimental design: Rats were obtained from the Basic Medical Sciences Animal House, University of the West Indies, Mona. Healthy male and female Wistar mixed breed rats were used within the weight range of 250-350 grams. The rats were kept in separate cages according to their sex to eliminate the possibility of impregnation. The rats were fed a daily diet of Purina Lab Chow and water administered ad libitum. All procedures were approved by and conducted in accordance with the guidelines of The University of the West Indies Animal Care and Use Committee.

Sample preparation: A dosage of $12.5 \mathrm{mg} \mathrm{kg}^{-1} \mathrm{BW}$ of captopril, CapSNO, SNAP and $30 \mathrm{mg} \mathrm{kg}^{-1} \mathrm{BW}$ of cysteine were used for the analysis. Saline $(0.4 \mathrm{~mL}$, $0.9 \% \mathrm{NaCl}$ ) was used to dissolve captopril and CapSNO and dimethyl sulfoxide [(DMSO, $0.4 \mathrm{~mL}$, $50 \%$ ); Sigma Chemical Co, St. Louis, MO, USA] was used to dissolve SNAP just before the beginning of the analysis. The solution was then administered into the tail vein of the rat immediately after the first blood sample was obtained for analysis.

Oral Glucose Tolerance Test (OGTT): Oral glucose tolerance (OGTT) was carried out using an automated method. The glucometer (Miles Inc. Diagnostics Division, Indiana, USA) was calibrated with standard solutions before use to allow for optimum performance. The test was carried out on rats to determine the effect of captopril, SNAP (Sigma Chemical Co, St. Louis, MO, USA), CapSNO, cysteine, CapSNO coadministered with cysteine and SNAP co-administered with cysteine, via intravenous administration on blood glucose levels in normoglycemic rats. S-nitrosocaptopril was synthesized as previously described ${ }^{[17]}$. Captopril, CapSNO and SNAP were administered at a dosage of $12.5 \mathrm{mg} \mathrm{kg}^{-1}$ body weight (BW) and cysteine was administered at a dosage of $30 \mathrm{mg} \mathrm{kg}^{-1} \mathrm{BW}$. Rats were fasted for approximately $15 \mathrm{~h}$, during this time only water was given ad libitum. A fasting blood sample at time (F1) was obtained from the rat's tail, immediately after which CapSNO, SNAP or captopril was administered via the intravenous route. Fasting blood samples were taken at F1, F2 and $0 \mathrm{~h}$. In experiments involving the co-administration of CapSNO and cysteine or SNAP and cysteine, the mixture was also administered after the blood sample was taken at F1. Immediately after the $1 \mathrm{~h}$ fasting sample was taken $(0 \mathrm{~h})$, a glucose load at a dosage of $1.75 \mathrm{~g} \mathrm{~kg}^{-1} \mathrm{BW}$ was then administered orally, after which postprandial blood samples were taken at $0.5 \mathrm{~h}$ intervals for a further $2.5 \mathrm{~h}$.

Total nitrite analysis: Total nitrite $\left(\mathrm{NO}_{2}^{-}\right)$ concentration was determined using a colourimetric non-enzymatic nitric oxide assay kit (Oxford Biomedical) which incorporates the use of the Greiss reagent. A dosage of $12.5 \mathrm{mg} \mathrm{kg}^{-1} \mathrm{BW}$ of captopril, CapSNO, SNAP and $30 \mathrm{mg} \mathrm{kg}^{-1} \mathrm{BW}$ of cysteine were administered via i.v. into the tail vein of the rat. After $30 \mathrm{~min}$ the rat was anaesthetized using a mild anesthetic and dissection carried out. Whole blood was carefully obtained from the heart and was placed in labeled $3 \mathrm{~mL}$ purple top $\mathrm{K}_{3}$-vacutainers. Centrifugation then followed at $3500 \mathrm{rpm}$ for $10 \mathrm{~min}$. Plasma $(1 \mathrm{~mL})$ was placed in labeled eppendorf tubes and stored at $-80^{\circ} \mathrm{C}$ for further 
Am. J. Biochem. \& Biotech., 4 (3): 265-272, 2008

use. The rat was then disposed of according to regulations stipulated in the animal care protocol.

Statistics: Each point was expressed as mean \pm standard error or the mean (SEM). For OGTT a minimum sample size of 9 rats was used to carry out the investigation. Blood samples were taken in duplicates for each time interval. For the total nitrite analysis, each compound was assayed in duplicates. In addition, each experiment was repeated three times. The average absorbance was determined from a standard curve and the necessary calculations done. The values were compared using Student's t-test. p-values less than 0.05 were considered to be significant in all cases.

\section{RESULTS AND DISCUSSION}

S-nitroso-captopril and SNAP had significant effects on the fasting blood glucose concentrations with values at $\mathrm{F} 2$ of $5.11 \pm 0.08$ and $5.91 \pm 0.11 \mathrm{mmol} \mathrm{L}^{-1}$ $(\mathrm{p}<0.05)$ respectively, compared with $3.80 \pm 0.23$ and $4.14 \pm 0.11 \mathrm{mmol} \mathrm{L}^{-1}$ in rats treated with saline and captopril respectively (Fig. 1). Rats treated with CapSNO had the highest postprandial blood glucose concentration of $6.81 \pm 0.23 \mathrm{mmol} \mathrm{L}^{-1}$ at the $0.5 \mathrm{~h}$ time point compared with $6.31 \pm 0.20$ and $5.92 \pm 0.14 \mathrm{mmol}$ $\mathrm{L}^{-1}$ in rats treated with SNAP and captoril respectively. These values were significantly different from the postprandial blood glucose concentration of rats treated with saline at the same time point $\left(4.58 \pm 0.29 \mathrm{mmol} \mathrm{L}^{-1}\right.$, $\mathrm{p}<0.00001)$. There was a gradual decline in mean blood glucose concentrations from peak values in rats treated with CapSNO, SNAP or captopril. The blood glucose concentrations in rats which were treated with SNAP or CapSNO were $5.46 \pm 0.12$ and $5.26 \pm 0.05 \mathrm{mmol} \mathrm{L}^{-1}$ respectively at the $2.5 \mathrm{~h}$ time point compared with $4.63 \pm 0.12 \mathrm{mmol} \mathrm{L}^{-1}$ in rats treated with captopril $(\mathrm{p}<0.05)$.

Rats treated with cysteine only and CapSNO and cysteine showed lower postprandial blood glucose concentrations at the $0.5 \mathrm{~h}$ time point $(6.24 \pm 0.29$ and $6.10 \pm 0.15 \mathrm{mmol} \mathrm{L}^{-1}$ respectively, Fig. 2) compared with rats treated with CapSNO only $(6.81 \pm 0.23 \mathrm{mmol}$ $\left.\mathrm{L}^{-1}\right)$. The blood glucose concentration in rats treated with CapSNO and cysteine decreased slightly at the 1.0 $\mathrm{h}$ time point and then gradually increased to peak value at the $2.5 \mathrm{~h}$ time point. This trend is in contrast to the postprandial blood glucose concentration in rats treated with cysteine only which increased slightly at the $1.0 \mathrm{~h}$ time point and then gradually decreased with the lowest

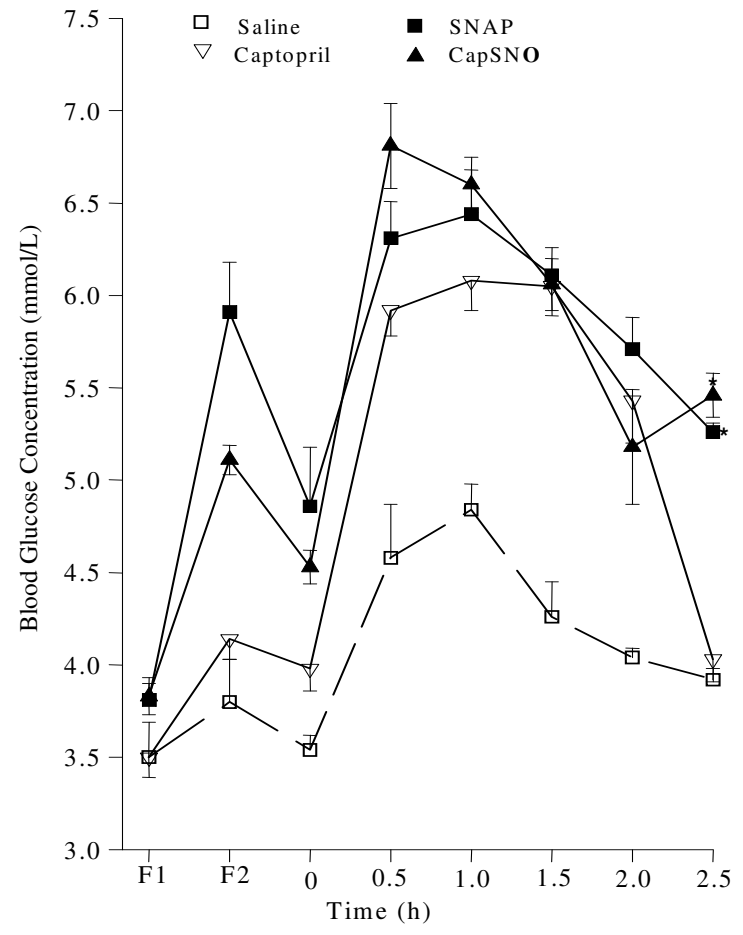

Fig. 1: Effect of captopril, SNAP and CapSNO on blood glucose concentration

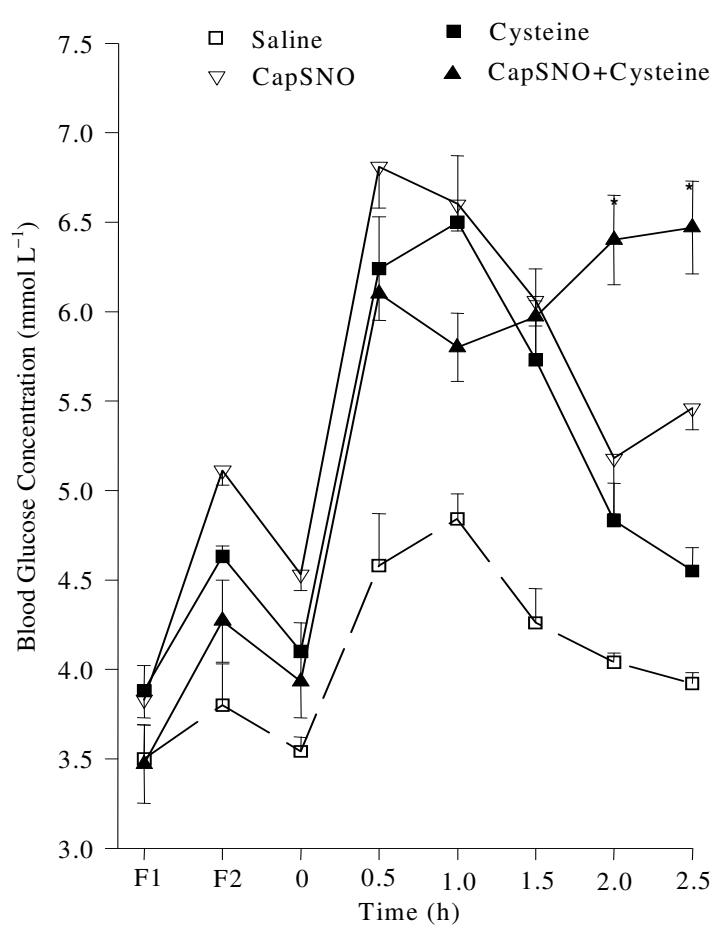

Fig. 2: The Effect of CapSNO co-administered with cysteine on Blood Glucose Concentration 
Am. J. Biochem. \& Biotech., 4 (3): 265-272, 2008

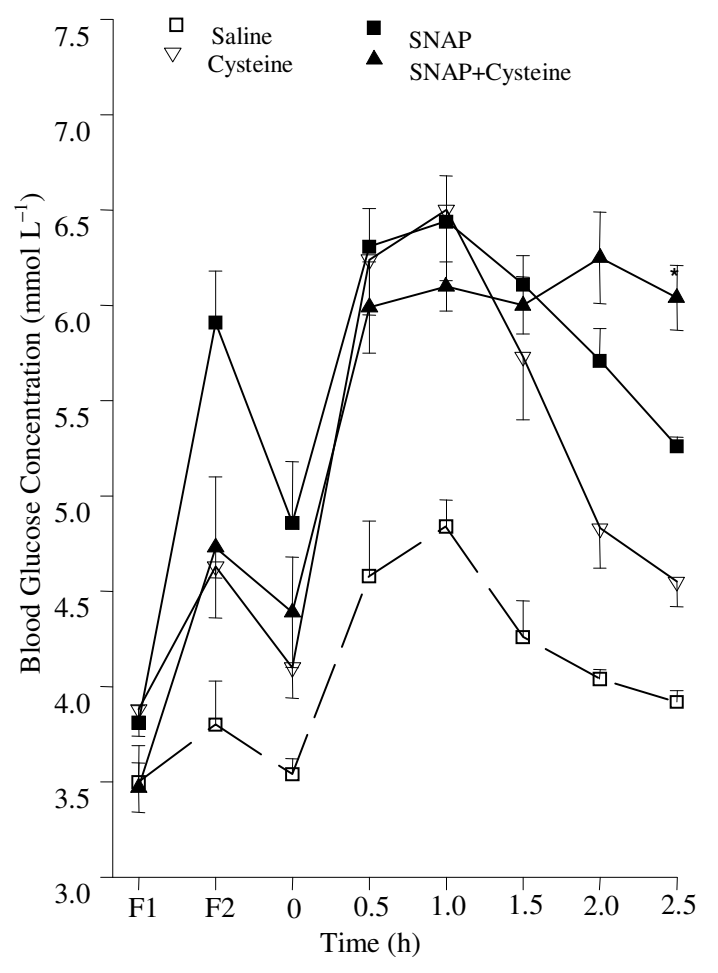

Fig. 3: The Effect of SNAP co-administered with cysteine on Blood Glucose Concentration

value at the $2.5 \mathrm{~h}$ time point. There was significant difference in postprandial blood glucose concentrations at the 2.0 and $2.5 \mathrm{~h}$ time points in rats co-administered with CapSNO and cysteine giving values of $6.40 \pm 0.25$ and $6.47 \pm 0.26 \mathrm{mmol} \mathrm{L}^{-1}$ respectively compared with those treated with only CapSNO with values of $5.18 \pm 0.31 \mathrm{mmol} \mathrm{L}^{-1}(2.0 \mathrm{~h})$ and $5.46 \pm 0.11 \mathrm{mmol} \mathrm{L}^{-1}$ $(2.5 \mathrm{~h}, \mathrm{p}<0.05)$. There were also significant differences in postprandial blood glucose concentrations in rats treated with CapSNO observed at the $2.5 \mathrm{~h}$ time points and those treated with cysteine $(4.55 \pm 0.13, \mathrm{p}=0.003)$.

The postprandial blood glucose concentrations of rats treated with SNAP, or cysteine were slightly higher than those of rats co-administered with SNAP and cysteine at the 0.5 and $1.0 \mathrm{~h}$ time points (Fig. 3). There was a gradual decrease thereafter in the blood glucose concentrations in rats treated with SNAP or cysteine, with the lowest values observed at the $2.5 \mathrm{~h}$ time points (i.e., $5.26 \pm 0.05$ and $4.55 \pm 0.13 \mathrm{mmol} \mathrm{L}^{-1}$ respectively). This is in contrast with the postprandial blood glucose concentration in rats treated with both SNAP and cysteine which showed an increase to a peak value at the $2.0 \mathrm{~h}$ time point $\left(6.25 \pm 0.24 \mathrm{mmol} \mathrm{L}^{-1}\right)$ and then decreased slightly at the $2.5 \mathrm{~h}$ time point $\left(6.04 \pm 0.17 \mathrm{mmol} \mathrm{L}{ }^{-1}, \mathrm{p}<0.05\right)$. Therefore, significant

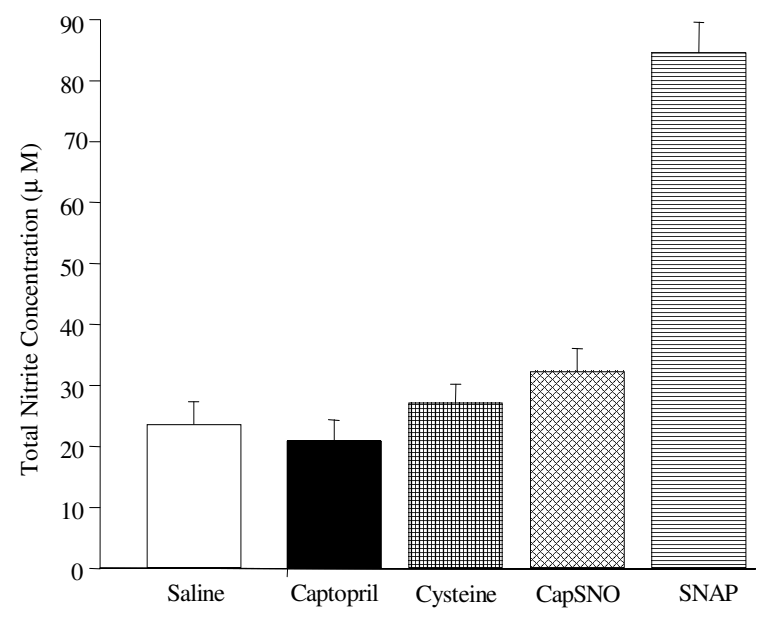

Fig. 4: The Effect of CapSNO, SNAP and Captopril on Total Nitrite Concentration

differences in postprandial blood glucose concentration were observed between rats treated with cysteine or SNAP compared with those co-administered with both SNAP and cysteine at the $2.0 \mathrm{~h}(\mathrm{p}<0.05)$ and $2.5 \mathrm{~h}$ time points $(\mathrm{p}<0.05)$.

The total nitrite concentration in rats that were administered with saline was $23.61 \pm 3.75 \mu \mathrm{M}$, which represented endogenous nitrite production (Fig. 4). Captopril-treated rats gave a total nitrite concentration of $20.90 \pm 3.45 \mu \mathrm{M}$. On the other hand CapSNO $(32.36 \pm 3.66 \mu \mathrm{M})$ and SNAP $(84.57 \pm 5.68 \mu \mathrm{M})$ treated rats showed a significant increase in the total nitrite concentration when compared with either captopril or saline treated rats $(\mathrm{p}<0.05)$. In addition rats that were administered with SNAP showed a more significant increase in total nitrite concentration than rats treated with CapSNO ( $\mathrm{p}=0.02)$.

The data showed that there was an increase in the total nitrite concentration to $52.63 \pm 2.69 \mu \mathrm{M}$ for CapSNO and cysteine-treated rats compared to rats administered with only CapSNO $(32.36 \pm 3.66 \mu \mathrm{M}, \mathrm{p}=$ 0.03, Fig. 5).

In this study the nitric oxide donors SNAP and CapSNO caused impaired glucose tolerance in normoglycemic rats by increasing fasting and even more significantly postprandial blood glucose concentrations. However, the major finding of the study is that the co-administration of cysteine and SNAP, or cysteine and CapSNO in the rats increased the hyperglycemic effect at the 2.0 and $2.5 \mathrm{~h}$ time points, resulting in further deterioration of glycemic control. The post-prandial blood glucose concentration at these two time points are significantly higher than those obtained upon administration only SNAP or 


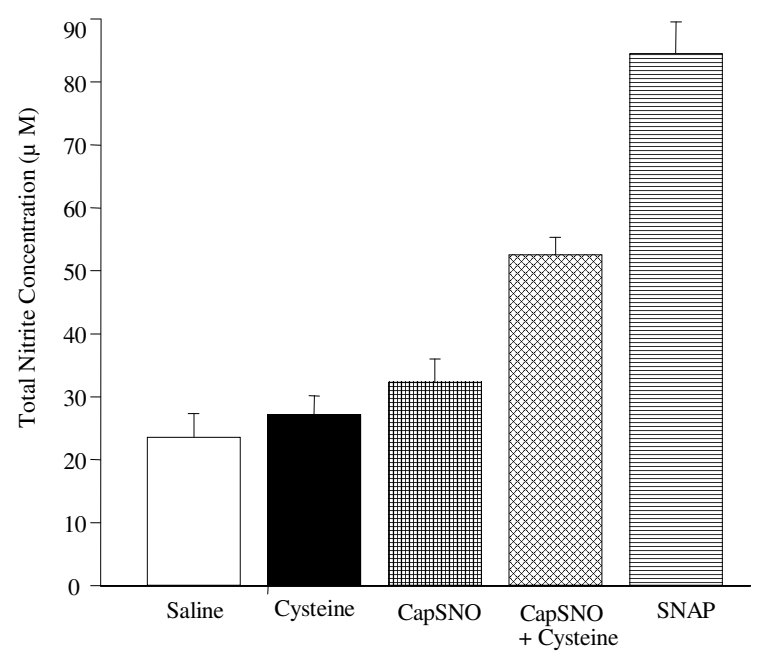

Fig. 5: The effect of CapSNO or SNAP coadministered with Cysteine on Total Nitrite Concentration

CapSNO only. The hyperglycemic effect of SNAP and CapSNO are in agreement with previous reports by McGrowder and colleagues who showed that GSNO and SNAP increased postprandial blood glucose concentration in normoglycemic dogs. These investigators showed decreased postprandial plasma insulin concentrations ${ }^{[12]}$ and increased plasma glucagon concentration ${ }^{[18]}$. The cysteine administered deteriorates the decreased glucose tolerance by the NO donors and may delay or decrease insulin response to a glucose challenge in an animal model, thereby prolonged the postprandial hyperglycemia ${ }^{[12]}$.

S-nitroso-N-acetylpenicillamine and CapSNO are primary and tertiary RSNOs respectively, however due to the close molecular weight of the compounds; there would not be any significant difference in the rate of diffusion through the vascular system. S-nitrosocaptopril having a more stable structure would be more resistant to decomposition when compared with $\mathrm{SNAP}^{[9]}$. The ability of SNAP to permeate cell membrane has not been fully elucidated, however it has been shown that CapSNO is able to permeate these barriers $^{[6]}$. The increase in blood glucose concentrations was more significant in SNAP-treated rats when compared with CapSNO treated rats. The hyperglycemic effect observed may be linked to the potency of SNAP as well as the ease at which this compound is able to decompose in vivo by a mechanism that is facilitated by endogenous reduced metal ions and thiols. Nitric oxide released from SNAP and CapSNO may be toxic at non- physiological concentration where it appears to function as a cellular effector molecule that mediates both cytostatic and cytotoxic effects ${ }^{[16]}$. Previous evidence have indicated that pancreatic islet cells exposed to the NO-donor streptozotocin caused lasting, damage to the $\beta$-cells, characterized by a persistent impairment in glucose metabolism and a defective insulin response ${ }^{[19]}$. The mechanism by which streptozotocin affects the pancreas could account for the hyperglycemic effect observed in the rats treated with SNAP or CapSNO.

S-nitrosocaptopril and SNAP were used in our experiments because these two compounds are the most typical and stable S-nitrosothiols and have been widely used in the biological research on NO. The interactions of NO with sulfhydryl-containing molecules and enzymes have gained considerable importance ${ }^{[5,6]}$. In many biological systems, S-nitrosylation reactions, transferring NO from CapSNO and SNAP to a protein sulfhydryl group, affect protein function. The blood glucose concentrations for cysteine and SNAP or cysteine co-administered with CapSNO-treated rats were well outside of the normal limits at the 2.0 and 2.5 $\mathrm{h}$ time points and were significantly elevated above that of their counterparts treated with only SNAP or CapSNO $(\mathrm{p}<0.05)$ suggesting further deterioration of glucose tolerance. The addition of cysteine to SNAP, or CapSNO increased postprandial blood glucose concentration possibly by increasing the efficacy of NO release. The effect observed when the RSNOs are coadministered with cysteine showed that decomposition via the transnitrosaton mechanism can occur in vivo using a high concentration of the thiol. In addition the result suggests that SNAP was less stable towards this type of decomposition. Therefore the decomposed product, NO and RSNOs are responsible for the observed hyperglycemic effect ${ }^{[12]}$.

Glutathione (GSH) is a critical intracellular reductant that functions in protecting cells from free radicals, reactive oxygen species and toxic substances. The intracellular concentration of GSH is in the range of 1-10 $\mathrm{mM}$ in many cells and is maintained by controls on its biosynthesis ${ }^{[20]}$. A number of mechanisms are available for transport of cystine or cysteine equivalents (cysteinylglycine) into cells. Cysteine can be taken up directly via the $\mathrm{Na}^{+}$- dependent amino acid transport system called the ASC system ${ }^{[21]}$. In many cells, this is a major route for supplying intracellular cysteine to maintain GSH levels. Rats treated with cysteine showed a hyperglycemic effect. S-Nitrosation of cysteine residues, resulting from the transfer of the oxidized form of $\mathrm{NO}$, to the - $\mathrm{SH}$ group of cysteine is another important mechanism in NO signaling. This mechanism may result in long-lasting effects due to posttranslational protein modification ${ }^{[22]}$. In arteries, protein $\mathrm{S}$-nitrosation is increased by exposure to exogenous NO or stimulation of endothelial NO synthase activity ${ }^{[23]}$. The increased amount of cysteine administered 
provides more thiol groups that can be S-nitrosated by endogenous and/or exogenous NO with the formation of S-nitrosocysteine. The biologically active NO subsequently released from s-nitrosocysteine may be responsible for the hyperglycemic effect observed, as this compound has been shown to have a shorter half life than CapSNO ${ }^{[16]}$ and SNAP ${ }^{[13]}$. Cysteine was shown to enhance the effect of SNAP, which is used commonly as an NO donor in in vitro experiments with intact cultured cells. It was suggested that cysteine could act by promoting transnitrosation from SNAP to S-nitrosocysteine, which would be more susceptible to intracellular decomposition, leading to more effective storage or transport of $\mathrm{NO}$ within the cell ${ }^{[9]}$.

The hyperglycemic effect of SNAP and CapSNO observed in the normoglycemic rats could arise as a result of a number of factors, including (i) inhibition of the secretory process by NO, such that insufficient insulin is secreted in response to a glucose challenge; (ii) a decrease in the vascular permeability of cell membrane by NO, resulting in decreased glucose and insulin delivery to the tissues; (iii) the action of NO released from SNAP and CapSNO on the pancreatic islet cells, impairing the first or early phase of the glucose-stimulated insulin release into the blood and (iv) NO inhibition of a number of crucial enzymes in the glycolytic pathway and electron chain such as glyceraldehyde-3-phosphate dehydrogenase (GAPDH) and aconitase respectively resulting in a decrease in glycolysis and glucose oxidation ${ }^{[24]}$. Of these mechanisms proposed, it is believed that the inhibitory effect of NO on GAPDH plays a significant role in the observed hyperglycemic effect. S-nitroso-Nacetylpenicillamine and GSNO have been found to inhibit the activity of GAPDH in skeletal muscles of type 2 diabetic rats ${ }^{[25]}$. Studies have also shown that the cysteine residue located at the active site of GAPDH (Cys-149) is oxidized by free radicals to a thiyl radical, which reacts with the neighboring coenzyme to form Cys-NAD $(H)$ linkages ${ }^{[26]}$. Inhibition of GAPDH is due primarily to the S-nitrosylation and, to a smaller extent, to the $\mathrm{NAD}(\mathrm{H})$ - dependent modification of Cys-149. SNAP has been found to induce the formation of the GAPDH thiyl radical only in the presence of L-cysteine and GAPDH thiyl radical was formed by SNAP only in the presence of oxygen ${ }^{[27]}$. The ability of peroxynitrite to oxidize protein thiols to thiyl radicals has been reported previously ${ }^{[27]}$. However, this oxidant in its anionic form is known to induce further oxidations of thiols to sulfinic or sulfonic acids ${ }^{[28]}$. The hyperglycemic effect observed in the rats could be due to a lesser extent on the inhibition of aldose reductase in the polyol pathway by NO released from SNAP and CapSNO. Nitric oxide released from NO donors has been found to inhibit aldose reductase in vascular smooth muscles by reversible S-thiolation and nitrosated at Cys-298 at the active site of the enzyme $^{[29]}$.

The inhibitory effect of NO could also conceivably be exerted through the formation of S-nitrosothiols, thereby impairing thiols groups essential for insulin action. The inhibitory effect of intracellular and extracellular NO on insulin secretory mechanisms is exerted through S-nitrosylation and hence the derangement of the reduced/oxidized glutathione balance, which previously has been shown to be of utmost importance for glucose-induced insulin secretion ${ }^{[30]}$. The evidence in favor of the role of exogenous NO in islet cell toxicity includes the lysis of islet cells in a concentration- and time-dependent manner, when they were exposed to the NO donor, sodium nitroprusside (SNP) ${ }^{[31]}$. In addition, SNP inhibits insulin-stimulated rates of glycogen synthesis at concentrations greater than $5 \mathrm{mM}$ in isolated rat soleus muscle preparations ${ }^{[32]}$. Experiments performed with SNAP, to validate the significance of $\mathrm{NO}$ in the development of diabetes, demonstrated cleavage of the DNA into nucleosomal fragments. It is proposed that internucleosomal DNA cleavage induced by exogenous NO activates poly(ADP ribose) synthase, which in turn depletes intracellular nicotinamide adenine dinucleotide (NAD) and causes poly-ADP-ribosylation of nuclear proteins, resulting in the deterioration of $\beta$-cell function ${ }^{[33]}$. Other supporting evidence includes a study done by Mosen and colleagues in which the intracellular NO donor hydroxylamine, suppressed glucose-induced insulin release and enhanced glucagon releas ${ }^{[33]}$. A possible mechanism for NO-induced inhibition of glucose-stimulated insulin release is exerted through a NO-mediated inactivation of the islet lysosomal enzyme acid glucan-1,4- $\alpha$-glucosidase activity $^{[34]}$.

The excretory form of $\mathrm{NO}$ is usually in the form of nitrates $\left(\mathrm{NO}_{3}^{-}\right)$and nitrites $\left(\mathrm{NO}_{2}^{-}\right)^{[35]}$ and the conversion of one form to the next readily takes place in vivo. However the total nitrite concentration incorporates both $\mathrm{NO}_{2}{ }^{-}$and $\mathrm{NO}_{3}{ }^{-}$species. The results from the total nitrite analysis showed that cysteine, or captopril did not cause a significant increase in the $\mathrm{NO}_{2}{ }^{-}$ concentration in vivo. This was expected as both thiols do not contain a nitro group in their structure. In addition the probability of a transnitrosation reaction occurring between these thiols and endogenous RSNOs is not expected to be significant since the latter group of compounds is present at low concentrations ${ }^{[36]}$ and is rapidly oxidized to other species ${ }^{[37]}$. However, these endogenous s-nitroso compounds have been shown to be very potent ${ }^{[38]}$. A significant increase in total nitrite 
concentration was observed when SNAP or CapSNO were administered. Rats treated with SNAP showed a higher total nitrite concentration than those administered with CapSNO. Therefore SNAP was able to decompose more readily in vivo than CapSNO. The decomposition of these compounds caused an increase in NO concentration which may have been responsible for the observed hyperglycemic effect. CapSNO caused a significant increase in total nitrite concentration when compared with saline treated rats. Elevated levels of plasma nitrate levels, assessed as NO production, were observed in cysteine and CapSNO treated-rats indicating enhanced NO release. This was evident by an increase in plasma nitrite concentration. The prolonged and exacerbated hyperglycemic effect in rats administered with CapSNO and cysteine could be explained in terms of the fact that exposure of the $\beta$ cells to increased NO levels resulted in further deterioration in $\beta$-cell function characterized by impairment in glucose metabolism and possible defective insulin response ${ }^{[39]}$. To ensure that plasma nitrite concentration reliably mirrored NO formation by the constitutive isoenzyme nitric oxide synthase, the dietary intake of nitrate was standardized, because the nitrate content of food varies widely. Dietary intake was standardized by giving each rat a defined diet daily.

\section{CONCLUSION}

In conclusion, the findings of this study indicate that NO released from SNAP and CapSNO elevated postprandial blood glucose and reduced glucose tolerance in normoglycemic rats. Cysteine exacerbated the decreased glucose tolerance by SNAP or CapSNO. To our knowledge, this is the report of the detrimental effect of cysteine on glucose metabolism in normoglycemic rats treated with $\mathrm{NO}$ donors and further current knowledge on the association of $\mathrm{NO}$ donors and thiols. An examination of the possible effect of SNAP and cysteine, or CapSNO and cysteine on glucoregulatory hormones in normoglycemic or diabetic rats is warranted.

\section{REFERENCES}

1. Beckman, J.S. and W.H. Koppenol, 1996. Nitric oxide, superoxide and peroxynitrite: The good, the bad and the ugly. Am. J. Physiol. Cell, 271: C1424-C1437.

2. Ignarro, L.J., 1990. Haem-dependent activation of guanylate cyclase and cyclic GMP formation by formation by endogenous nitric oxide: A unique transduction mechanism for transcellular signaling. Pharmacol. Toxicol., 67: 1-7.
3. Stamler, J.S., D.I. Simon, J.A. Osborne, M.E. Mullins, O. Jaraki, T. Michel, D.J. Singel and J. Loscalzo, 1992. S-nitrosylation of proteins with nitric oxide: Synthesis and characterization of biologically active compounds. Proc. Natl. Acad. Sci. USA, 89: 444-448.

4. Ignarro, L., H. Lippton, J.C. Edwards, W.H. Baricos, A.C. Hyman, P.J. Kodowitz and C.A. Gruetter, 1981. Mechanism of vascular smooth muscle relaxation by organic nitrates, nitrites, nitroprusside and nitric oxide: Evidence for the involvement of s-nitrosothiols as active intermediates. J. Pharmacol. Exp. Ther., 218: 739-749.

5. Kowaluk, E.A. and H. Fung, 1990. Spontaneous liberation of nitric oxide cannot account for in vitro vascular relaxation by s-nitrosothiols. J. Pharmacol. Exp. Ther., 255: 1256-1267.

6. Jia, L. and H. Wong, 2001. In vitro and in vivo assessment of cellular permeability and pharmacodynamics of s-nitrosylated captopril, a nitric oxide donor. J. Pharmacol., 134: 1697-1704.

7. Askew, S.C., D.J. Barnett, J. Mckninly and D.L. Williams, 1995. Catalysis of $\mathrm{Cu}^{+}$of nitric oxide release from s-nitrosothiols (RSNO). J. Chemical Soc. Perkins Trans., 2: 741-745.

8. Smith, J.N. and T. Dasgupta, 2000. Kinetics and mechanism of the decomposition of snitrosoglutathione by L-ascorbic acid and copper ions in aqueous solution to produce nitric oxide. Nitric Oxide: Biology and Chemistry, 4: 57- 66.

9. Singh, R.J., N. Hogg, J. Joseph and Kalyanaraman, 1996. Mechanism of NO release from Snitrosothiols. J. Biol. Chem., 271: 18596-18603.

10. Aquart, D.V. and T.P. Dasgupta, 2004. Dynamic interaction of vitamin $\mathrm{C}$ with some potent nitrovasodilators, SNAP and SNOCap, in aqueous solution. Biophys. Chem., 107: 117-131.

11. Williams, D.L., 1985. S-nitrosoation and reactions of S-nitroso compounds. Chem. Soc. Rev., 14: 171-196.

12. McGrowder, D., D. Ragoobirsingh and T. Dasgupta, 2001. Effects of s-nitroso-n-acetylpenicillamine administration on glucose tolerance and plasma levels of insulin and glucagon in the dog. Nitric Oxide: Biol. Chem., 5: 402-412.

13. Mathew, W.R. and S.W. Kerr, 1993. Biological activity of s-nitrosothiols: The role of nitric oxide. J. Pharmacol. Exp. Ther., 267: 1529-1537.

14. Al-Sa'doni, H. and A. Ferro, 2000. S-nitrosothiols: A class of nitric oxide-donor drugs. Clin. Sci., 98: 507-520. 
15. Shaffer, J.E., F. Lee, S. Thomson, H. Ba-Jin, J.P. Cooke and J. Loscalzo, 1990. The hemodynamic effects of S-nitrosocaptopril in anesthetized dogs. J. Pharmacol. Exp. Ther., 256: 704-710.

16. Lascalzo, J., D. Smick, N. Andon and J. Cooke, 1988. S-nitrosocaptopril: Molecular characterization and effects on the vasculature and on platelets. J. Pharmacol. Exp. Ther., 249: 726-729.

17. Nakae, I., M. Takahashi, T. Kinoshita, T. Matsumoto and M. Kinoshita, 1995. The effects of s-nitrosocaptopril on canine coronary circulation. J. Pharmacol. Exp. Ther., 274: 40-46.

18. McGrowder, D., D. Ragoobirsingh and T. Dasgupta, 1999. The hyperglycemic effect of S-nitrosoglutathione in the dog. Nitric oxide: Biology and Chemistry, 3: 481-491.

19. Turk, J., J.A. Corbett, S. Romanadham, A. Bohrer and M.L. McDaniel, 1993. Biochemical evidence for nitric oxide formation from streptozotocin in isolated pancreatic islets. Biochem. Biophys. Res. Commun., 197: 1458 - 1464.

20. Meister, A., 1995. Glutathione metabolism. Methods Enzymol., 251: 3-7.

21. Knickelbein, R.G., T. Seres, G. Lam, Jr R.B. Johnston and J.B. Warshaw, 1997. Characterization of multiple cysteine and cystine transporters in rat alveolar type II cells. Am. J. Physiol., 273 (Lung Cell Mo. Physiol. 17): L1147-L1155.

22. Stamler, J.S., S. Lamas and F.C. Fang, 2001. Nitrosylation. The prototypic redox-based signaling mechanism. Cell, 106: 675-683.

23. Gow, A.J., Q. Chen, D.T. Hess, B.J. Day, H. Ischiropoulos and J.S. Stamler, 2002. Basal and stimulated protein S-nitrosylation in multiple cell types and tissues. J. Biol. Chem., 277: 9637-9640.

24. Ishii, T., O. Sunami, H. Nakajima, H. Nishio, T. Takeuchi and F. Hata, 1999. Critical role of sulphenic acid formation of thiols in the inactivation of glyceraldehyde-3-phosphate dehydrogenase by nitric oxide. Biochem. Pharmacol., 58: 133-143.

25. McGrowder, D., K. Barrett, P. Brown and D. Ragoobirsingh, 2005. Exogenous nitric oxide inhibits glucose uptake by peripheral tissues in a diabetic rat model. Diabetologia Croatica, 34 (3): 77-85.

26. Minetti, M., A.M. Pietraforte, M. Di Stasi and C. Mallozzi, 1996. Nitric oxide-dependent NAD linkage to glyceraldehydes-3-phosphate dehydrogenase: Possible involvement of a cysteine thiyl radical intermediate. Biochem. J., 319: 369-375.
27. Gatti, R.M., R. Radi and O. Augusto, 1994. Peroxynitrite-mediated oxidation of albumin to the protein-thiyl free radical. FEBS Lett., 348: 287-290.

28. Radi, R., J.S. Beckman, K.M. Bush and B.A. Freeman, 1991. Peroxynitrite oxidation of sulfhydryls. The cytotoxic potential of superoxide and nitric oxide. J. Biol. Chem., 266: 4244-4250.

29. Ramana, K.V., D. Chandra, S. Srivastava, A. Bhatnagar and S.K. Srivastava, 2003. Nitric oxide regulates the polyol pathway of glucose metabolism in vascular smooth muscle cells. FASEB J., 17: 417- 425.

30. Panagiotidis, G., B. Akesson, E.L. Rydell and I. Lundquist, 1995. Influence of nitric oxide synthase inhibition, nitric oxide and hydroperoxide on insulin release induced by various secretagogues. Br. J. Pharmacol., 114: 289-296.

31. Burkart, V., K.D. Kronche, H.H. Brenner, H. Kolb and V. Kolb-Brachofen, 1991. Nitric oxide is a pathogenic factor in type 1 diabetes. Diabetologia 34(Suppl. 2): A95 [Abstract].

32. Young, M.E., G.K. Radda and B. Leighton, 1997. Nitric oxide stimulates glucose transport and metabolism in rat skeletal muscles in vitro. Biochem. J., 322: 223-228.

33. Zhang, J., V.L. Dawson and S.H. Synder, 1994. Nitric oxide activation of poly(ADPribose) synthetase in neurotoxicity. Science, 263: 687-689.

34. Mosen, H., A. Salehi and I. Lundquist, 1997. Islet acid glucan-1,4- $\alpha$-glucosidase in relation to nitric oxide and insulin secretion. Diabetologia, 41 (Suppl. 1): A 110 [Abstract].

35. Weitzberg, E. and J.O. Lundberg, 1998. Nonenzymatic NO production in humans. Nitric Oxide: Biology and Chemistry, 2: 1-7.

36. Tyurin, V.A., S. Liu, Y.Y. Tyurina, N.B. Sussman, C.A. Hubel, J.M. Roberts, R.N. Taylor and V.E. Kagan, 2001. Elevated Levels of snitrosoalbumin in preeclampsia plasma. Circulat. Res., 88: 1210-1215.

37. Gibson, Q.H. and F.J. Roughton, 1957. The kinetics and equilibria of the reactions of nitric oxide with sheep haemoglobin. J. Physiol., 136: 507-526.

38. Weinberger, B., D.L. Laskin, D. Heek and J. Laskin, 2001. The toxicology of inhaled NO. Toxicol. Sci., 59: 5-16.

39. Schmidt, H.W., T.D. Warner, K. Ishii, H. Sheng and F. Merad, 1992. Insulin secretion from pancreatic beta-cells caused by L-arginine-derived nitrogen oxides. Science, 255: 721-723. 